\title{
Ischemia of The Right Upper Limb in A Newborn With Down Syndrome A Case Report \\ Diouf Cheikh ${ }^{1 *}$, Ndoye Ndey Aby ${ }^{2}$, Kane Ahmed $^{3}$, Seye $\mathrm{Cheikh}^{4}$, Diallo Ibrahima ${ }^{5}$, Ndour Oumar ${ }^{6}$, Ngom Gabriel $^{7}$
}

\author{
${ }^{\mathrm{I}}$ Department of surgery UFRSS University Assane Seck / Ziguinchor regional Hospital Sénégal \\ ${ }^{2}$ Departement Department of Surgery Université Cheikh Anta diop / hopital d'enfants Albert Royer Dakar /senegal University of sciences and \\ technologies Nouakchott Mauritania \\ ${ }^{4}$ University Alioune Diop Bambey \\ ${ }^{5}$ Departement of surgery regional hospital of Ziguinchor Sénégal \\ ${ }^{6}$ Departement of surgery university cheikh Anta Diop Dakar Senegal \\ ${ }^{7}$ Departement of surgery university cheikh Anta diop
}

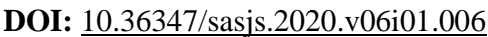

| Received: 10.01.2020 | Accepted: 17.01.2020 | Published: 29.01.2020

*Corresponding author: Dr. Cheikh Diouf

Abstract

Down syndrome is a chromosomal aberration described for the first time by J. Lejeune and al in 1959 . It is indeed the most frequent of the autosomal chromosomal anomalies and one of the main causes of intellectual disability. It is known by its association with several malformations, especially cardiac, digestive and urinary. Ischemia of a limb with no notion of trauma remains a clinical situation little reported in the literature. We report the case of a male newborn admitted for ischemic gangrene of upper extremity. Observation: A male newborn is admitted to the emergency room for functional impotence of the right upper limb. The examination found a Down's syndrome facies and ischemic gangrene of the right upper limb involving the hand and going up to the middle thirds of the right forearm. No notion of trauma to the limb was found during the interrogation. The Doppler ultrasound of the upper limb was in favor of an alteration in the flow of the right ulnar and radial arteries. The cardiac ultrasound showed a CIA with 3 mm ostium segundum, a persistence of an arterial canal. The hemoglobin level was $17 \mathrm{~g} / \mathrm{dl}$ and he had a leukocytosis at $13000 \mathrm{~g} /$ dl. The diagnosis of idiopathic ischemia of the upper limb on a trisomy 21 site was accepted. He underwent amputation of the forearm, the evolution of which is marked by "suppuration of the stump of the amputation. Conclusion: Idiopathic ischemia in Down's syndrome is a rarely encountered disease. Its early diagnosis and its appropriate management guarantee a good development. Its evolution sometimes leads to complications that can be life-threatening.

Keywords: Down's syndrome, ischemia, gangrene, amputation.

Copyright @ 2020: This is an open-access article distributed under the terms of the Creative Commons Attribution license which permits unrestricted use, distribution, and reproduction in any medium for non-commercial use (NonCommercial, or CC-BY-NC) provided the original author and source are credited.

\section{INTRODUCTION}

Trisomy 21 is a chromosomal aberration described for the first time by J. Lejeune and al in 1959 [1]. It represents the most frequent autosomal chromosomal abnormalities. It can be associated with several malformations which can be cardiac, digestive and urinary [1]. Ischemia of a limb on down syndrome without notion of trauma is not frequently reported in the literature. We report the case of a male newborn admitted for ischemic gangrene of the upper limb on trisomy 21 .

\section{OBSERVATION}

A male newborn born from a well-followed pregnancy with a vaginal delivery without any notion of inbreeding is received five days after born for cyanosis, functional impairment in the right upper limb. Symptoms began three days after birth in the phalanges of the right hand. The evolution was rapidly progressive reaching the forearm on the fourth day of life. The interrogation found no notion of even minor trauma in the upper limb. The physical examination identified a hemodynamically, neurologically and respiratory stable infant. He weighed $3350 \mathrm{~g}$, his temperature was 37.2 degrees Celsius. There was also an ischemic gangrene of the upper limb going up to half of the right forearm (Figure 1). The biological assessment showed a hemoglobin level of $17 \mathrm{~g} / \mathrm{dl}$, a hyperleukocytosis $13000 \mathrm{~g} / \mathrm{dl}$. The hemostasis balance was normal. The Doppler ultrasound of the right upper limb showed an alteration in the flow of the right ulnar and radial arteries. The cardiac ultrasound had objectified an atrial communication with ostium segundum of $3 \mathrm{~mm}$, a 
persistence of an arterial channel. He underwent amputation of the right forearm and antibiotic therapy based on amoxicillin clavulanic acid and analgesics. The evolution was marked by a suppuration of the stump of amputation (Figure 2). The bacteriology showed an Escherichia coli sensitive to ceftriaxone.

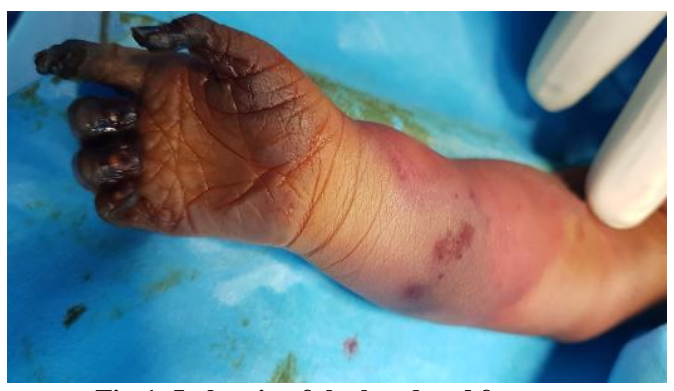

Fig-1: Ischemia of the hand and forearm

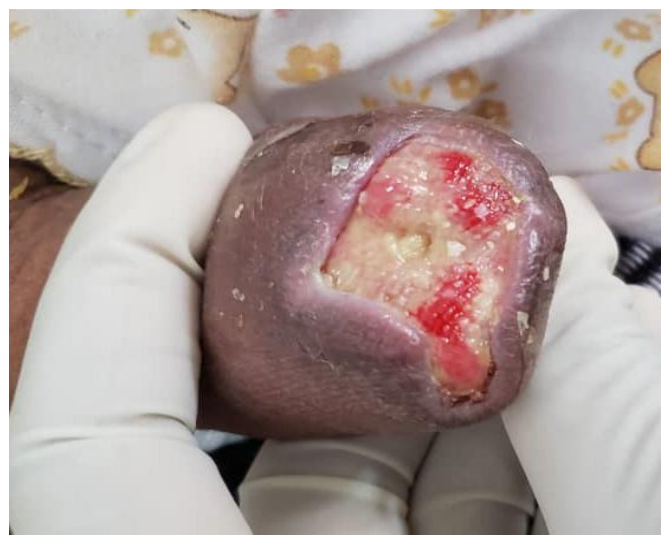

Fig-2: Suppuration of the amputation stump

\section{DISCUSSION}

Gangrene of the extremities of the newborn is a very rare clinical entity. Several phenomena are incriminated: thromboembolic, gestational diabetes, prematurity, congenital heart disease, obstetric trauma, polycythemia, septicemia... .etc. [2,3]. Its association with Down's syndrome can be favored by specific actors present during Down's syndrome:

proteins encoded on chromosome 21 (superoxide dismutase 1, cyctathionine b-synthase and collagen type 4 and the gamma interferon receptor) affect arterial physiology and may be involved in the narrowing of the cerebral arteries; autoimmunity has also been reported as a possible mechanism; the presence of anti-phospholipid antibodies which can give arterial thrombosis; anti thyroid microsome antibodies; the possibility of protein $\mathrm{C}$ deficiency, responsible for thromboembolic events [4].

In our patient Down's syndrome and heart disease could explain the rapidly progressive evolution of ischemia. The management of this pathology necessarily involves an early diagnosis at the ischemia stage is essentially based on the administration of heparin [1-7]. This early heparin therapy often allows a better evolution while avoiding aggressive amputations in this neonatal context. In some cases of early diagnosis at the ischemia stage, some authors discuss the value of surgical techniques for limb revascularization [5]. If the diagnosis is made at the stage of gangrene then amputation is necessary to avoid the many complications. However, this amputation should only be considered after the presence of a demarcation furrow as in our case [5-7]. Evolution is usually favorable however in our case we noted a suppuration of the amputation stump which could well be due to a nosocomial infection. The presence of an infection in this context requires an antibiogram rapidly in order to identify and readjust the antibiotherapy in the patient.

\section{CONCLUSION}

Idiopathic ischemia in the newborn is a condition rarely encountered. It requires early diagnosis and appropriate management to avoid limb amputations.

\section{CONFLICT OF INTEREST} this article

Authors point out no conflict of interest for

\section{REFERENCIES}

1. Salomon L J Diagnosis of Down's syndrome as simple as a blood test? Obstetrics and fertility gynecology. 41(2013) 77-79

2. Anass Ayyad, Sahar Messaoudi, Amrani. Congenital gangrene: a rare condition during the neonatal period: a case study The Pan African Medical Journal. 2019;33:59.

3. Chaanine A, Hugonenq C, Lena G, Mancini J. Neurological complications in Down syndrome. Archives de pediatrie: organe officiel de la Societe francaise de pediatrie. 2008 Apr;15(4):388-96.

4. Sfaihi LB, Ayedi A, Chaari W, Aloulou H, Kammoun T, Mnif Z, Mahfoudh A, Triki C, Hachicha M. Moyamoya and Down syndrome: about 2 cases. Archives de pediatrie: organe officiel de la Societe francaise de pediatrie. 2008 Jul;15(7):1197-200.

5. Matos JM, Fajardo A, Dalsing MC, Motaganahalli R, Akingba GA, Murphy MP. Evidence for nonoperative management of acute limb ischemia in infants. Journal of vascular surgery. $2012 \mathrm{Apr}$ 1;55(4):1156-9.

6. Zetlitz E, Weiler-Mithoff E, Turner T. Idiopathic neonatal ischemia in the upper limb: the role of the microsurgeon. American journal of perinatology. 2008 Sep;25(08):513-6.

7. Rachid Zaghloul, Comlan Mawuko Blitt, Hamid Jiber, Abdellatif Bouarhroum. Acute non-traumatic ischemia of the lower limb in a 1 year old infant: clinical case and review of the literature Pan Afr Med Journal. 2015; 21:171. 